\title{
Novel Nonlinear Magneto-Optical Effects
}

\author{
A. Kirilyuk and Th. Rasing \\ Research Institute for Materials, University of Nijimegen, 6525 ED Nijimegen, The Netherlands \\ V.V. Pavlov and R.V, Pisarev \\ Ioffe Physico-Technical Institute of the Russian Academy of Sciences, 194021 St. Petersburg, Russia
}

In the area of magnetism, optical methods have always been very effective. As a consequence, magneto-optics now serves as one of the main tools to study thin magnetic films, multilayers and surfaces. In addition to the well known linear magneto-optical techniques, Magnetizationinduced Second Harmonic Generation (MSHG) is a rapidly developing new technique, capable to probe surfaces and buried interfaces with submonolayer sensitivity. It appears that the magneto-optical effects are strongly enhanced in the nonlinear case compared to their linear equivalents. On the other hand, due to the more complicated form of the nonlinear optical tensor, essentially new effects become observable ${ }^{1,2}$.

Here we present a comprehensive classification of the nonlinear magneto-optical effects, both theoretically from an analysis of the point group symmetry and experimentally using the example of thin magnetic garnet films. The nonlinear optical tensor is considered as expansion up to the linear in magnetization term: $\chi^{(2)}=\chi_{0}^{(2)}+\chi_{1}^{(2)} \cdot \mathbf{M}$. Several new magneto-optical effects that have no equivalent in the linear case are predicted and observed, such as a transversal effect linear in $\mathbf{M}$, a longitudinal effect of linear magnetic dichroism, nonreciprocal with respect to the light helicity circular magnetic dichroism, etc. A close relation of these effects with the crystallographic anisotropy of the sample will certainly favour the use of this technique as a powerful method to study the relation between structure and magnetism.

${ }^{1}$ V. V. Pavlov et al., Phys. Rev. Lett. 78, 2004 (1997).

${ }^{2}$ R.V. Pisarev, V.V. Pavlov, A. Kirilyuk and Th. Rasing, J. Magn. Soc. Jpn. 20, 23-28 (1996). 